\title{
Haptoglobin Duplicon, Hemoglobin, and Vitamin C: Analyses in the British Women's Heart and Health Study and Caerphilly Prospective Study
}

\author{
Philip A. I. Guthrie, ${ }^{1}$ Mohammad R. Abdollahi, ${ }^{1}$ Tom Gaunt, ${ }^{1,2}$ \\ Debbie A. Lawlor, ${ }^{2}$ Yoav Ben-Shlomo, ${ }^{3}$ John Gallacher, ${ }^{4}$ George Davey Smith, ${ }^{2}$ \\ Ian N. M. Day, ${ }^{1}$ and Santiago Rodriguez ${ }^{1}$ \\ ${ }^{1}$ Bristol Genetic Epidemiology Laboratory, School of Social and Community Medicine, University of Bristol, \\ Oakfield House, Oakfield Grove, Bristol BS8 2BN, UK \\ ${ }^{2}$ MRC Integrative Epidemiology Unit (IEU), University of Bristol, Bristol BS8 2BN, UK \\ ${ }^{3}$ School of Social and Community Medicine, University of Bristol, Bristol BS8 2PS, UK \\ ${ }^{4}$ Institute of Primary Care \& Public Health, Cardiff University School of Medicine, Cardiff CF14 4XN, UK
}

Correspondence should be addressed to Philip A. I. Guthrie; philip.guthrie@bristol.ac.uk

Received 21 August 2014; Revised 31 October 2014; Accepted 7 November 2014; Published 30 November 2014

Academic Editor: Seul-Ki Jeong

Copyright @ 2014 Philip A. I. Guthrie et al. This is an open access article distributed under the Creative Commons Attribution License, which permits unrestricted use, distribution, and reproduction in any medium, provided the original work is properly cited.

Background. Haptoglobin acts as an antioxidant by limiting peroxidative tissue damage by free hemoglobin. The haptoglobin gene allele $\mathrm{Hp} 2$ comprises a $1.7 \mathrm{~kb}$ partial duplication. Relative to allele $\mathrm{Hp} 1, \mathrm{Hp} 2$ carriers form protein multimers, suboptimal for hemoglobin scavenging. Objective. To examine the association of haptoglobin genotype with a range of phenotypes, with emphasis on vitamin C and hemoglobin levels. Methods. We applied a quantitative PCR assay for the duplication junction to two population cohorts including 2747 British women and 1198 British men. We examined the association of haptoglobin duplicon copy number with hemoglobin and vitamin $\mathrm{C}$ and used the copy number to complete a phenome scan. Results. Hemoglobin concentrations were greater in those with Hp2,2 genotype, in women only (Hp1,1 $13.45 \mathrm{~g} / \mathrm{dL}, \mathrm{Hp1}, 213.49 \mathrm{~g} / \mathrm{dL}, \mathrm{Hp} 2,213.61 \mathrm{~g} / \mathrm{dL} ; P=0.002)$, though statistically there was no evidence of a difference between the sexes $(z$ value $=1.2, P=0.24)$. Haptoglobin genotype was not associated with vitamin $\mathrm{C}$ or any other phenotype in either cohort. Conclusions. Our results do not support association of haptoglobin genotype with vitamin $\mathrm{C}$ or with other phenotypes measured in two population cohorts. The apparent association between haptoglobin genotype and hemoglobin in the women's cohort merits further investigation.

\section{Introduction}

The haptoglobin gene HP exists in the human population in two forms: Hp1 and Hp2 (throughout this paper the classic haptoglobin duplicon nomenclature will be used, i.e., Hp1 refers to the allele without the duplication and $\mathrm{Hp} 2$ refers to the allele with duplication). Hpl is the less frequent allele in Europeans. Hp1,1 refers to the homozygote for the Hp1 allele, and $\mathrm{Hp} 2,2$ refers to the homozygote for the $\mathrm{Hp} 2$ allele. Exons 5 and 6 of $\mathrm{Hp} 2$ represent duplication of a $1.7 \mathrm{~kb}$ segment containing exons 3 and 4 of Hp1 [1]. The protein product for $\mathrm{Hp} 1,1$ forms a dimer, whereas the products of $\mathrm{Hp} 1,2$ or $\mathrm{Hp} 2,2$ are multimers of varying size and complexity, greater in the Hp2,2 genotype. The principal function of haptoglobin, which is normally present at a greater than 400 -fold molar excess compared with free hemoglobin [2], is to scavenge free hemoglobin [3] which has been liberated into the plasma by intravascular haemolysis. The haptoglobin/hemoglobin complex is cleared from the bloodstream by circulating monocytes or in the liver by Kuppfer cells, and the heme iron is recycled. At sites of tissue damage, the complex is cleared by macrophages.

The range of possible forms of haptoglobin, from dimer to large multimer depending on genotype, results in a gene 
product with a range of properties and potentially complex interactions. For example, although the hemoglobin-binding capacity of Hp2,2 is lower than that of Hp1,1 [4], the Hp2,2hemoglobin complex binds to CD163 (the haptoglobin receptor expressed on macrophage and monocyte cell surfaces) with a 10-fold higher affinity than the Hp1,1-hemoglobin complex [5].

The aim of this study was to examine the association of haptoglobin genotype with a wide range of phenotypes. This is justified on the basis of the hypothesised antioxidant properties of haptoglobin and claims for the involvement of antioxidation in a wide range of health related outcomes. However, within this broad phenome scan, we had three specific prior hypotheses.

(1) Higher levels of vitamin C would be found in individuals not carrying the $\mathrm{Hp} 2$ allele because haptoglobin is involved in redox reaction, which should affect vitamin C levels [6] and, as described above, is intimately involved with the scavenging of circulating hemoglobin.

(2) Higher total levels of hemoglobin would be found in individuals carrying the Hp2 allele because of less efficient clearance. This hypothesis was based on the specific high affinity protein interaction of haptoglobin with hemoglobin and reports of haptoglobin genotype association with hemoglobin level in malaria [7].

\section{Materials and Methods}

We developed a novel high-throughput liquid-phase assay for detection of the $H p$ duplicon, based on fluorescence ratiometry between junction and reference polymerase chain reaction (PCR) amplicons using a melt curve analysis [8]. We used this assay to genotype participants in two British cohort studies: the British Women's Heart and Health Study (BWHHS) and the Caerphilly Prospective Study (CaPS) of men. Because of the possibility that copy number variation in $H p$ could have a wide range of effects, relating to its antioxidant properties, we completed a "phenome scan" in these two cohorts, examining a range of blood measures and clinical traits with genotype.

2.1. British Women's Heart and Health Study (BWHHS). BWHHS is a cohort of 4,286 women, who were randomly selected from 23 British towns and who were of the age between 60 and 79 years at enrolment (1999-2001) [9]. At baseline assessment the women completed a questionnaire, a nurse-led health interview, and a physical examination at which fasting blood samples (minimum 8 hours) were taken. Medical records were reviewed for all evidence of past history of cardiovascular disease, type 2 diabetes, and cancer. The women have been followed up with information on new disease events obtained from participant completed questionnaires and a two-year review of their medical records and with mortality data obtained from the UK National Health Service central register.
For further details of BWHHS, including analyte preparation, please see Supplementary Material available online at http://dx.doi.org/10.1155/2014/529456.

2.2. Caerphilly Prospective Study (CaPS). All men from Caerphilly, South Wales, and surrounding villages of age between 45 and 59 years were contacted and invited to take part in a health survey, resulting in 2512 (89\% response rate) men participating in the baseline examination that was undertaken between 1979 and 1983 (Phase I). Phase II (1984-1988) consisted of those from Phase I who had been recontacted, together with an additional group who had since moved into the study area (Phase II $n=2,398$ ). The examination consisted of a standard medical history, venepuncture for biochemical and hormonal assays, and physical examination including weight, height, blood pressure, and 12-lead ECG. Hemoglobin was measured by a modification of the Dacie and Lewis method using a dedicated hemoglobinometer. Vitamin C was not measured. Subjects were defined as having diabetes (type 2 unless otherwise specified) if they had been previously diagnosed by their general practitioner or if their fasting glucose concentrations were greater than or equal to $7.8 \mathrm{mmoL} / \mathrm{L}$. Other phenome variables were measured as described previously [10]. Of the participants for whom DNA was available, 1198 had complete data on the combination of $\mathrm{CHD}$, type 2 diabetes (based on self-reported diagnosis from questionnaires), and haptoglobin genotype, and so all our analyses were conducted on 1198 men.

2.2.1. Genotyping Assay. The $H p$ junction region and a reference sequence in TP53 not subject to copy number variation were coamplified by PCR. Reactions were performed using 20 ng genomic DNA as template in 384-well plates. Following 33-cycle PCR optimised to preserve ratio of amplification of test and reference, the ratio of test to reference was determined by fluorescence quantification of Eva Green binding during real time melting of the amplicons in a 384-well LightTyper (Roche Diagnostics); test and reference amplicons were designed to melt at different temperatures [8]. Validations included typing of genotype known DNAs (gift of Dr. Werner Koch (German Heart Centre, Lazarettstrasse 36, D-80636, Munich) and Dr. Joris Delanghe (Department of Laboratory Medicine, University Hospital Ghent, Belgium)), comparison of our ratiometric assay with the assay described by Koch et al. [11], and evaluation of the cluster quality differentiating the clusters of ratios representing 1 or 2 copies of the $H P$ duplicon junction. The absence of a target amplicon first derivative fluorescence peak but presence of a reference peak signified zero copies of the duplicon junction.

\section{Statistics}

Linear (for continuous traits) and logistic (for binary traits) regression was used to examine the association of genotype with phenotypes. Where traits were positively skewed (triglycerides, glucose, insulin), we used a natural logarithm transformation in these regression analyses and then backtransformed for table presentation. The $P$ value, with Bonferroni correction, for the phenome scan of the BWHHS data 
TABLE 1: Haptoglobin genotype and allele frequency for BWHHS and CaPS cohorts.

\begin{tabular}{lcccc}
\hline \multirow{2}{*}{ Genotype/Allele } & \multicolumn{2}{c}{ Number (\%) [expected number] } \\
& \multicolumn{2}{c}{ BWHHS } & \multicolumn{2}{c}{ CaPS } \\
\hline Hp1,1 & $417(13.3)$ & {$[416.2]$} & $204(14.4)$ & {$[210.9]$} \\
Hp1,2 & $1447(46.3)$ & {$[1448.5]$} & $685(48.4)$ & {$[671.2]$} \\
Hp2,2 & $1261(40.3)$ & {$[1260.2]$} & $527(37.2)$ & {$[533.9]$} \\
Hp1 & $2281(36.5)$ & & $1093(38.6)$ \\
Hp2 & $3969(63.5)$ & $1739(61.4)$ \\
\hline \multicolumn{3}{c}{ BWHHS } & CaPS \\
\hline HWE chi ${ }^{2}, 1 \mathrm{df}(P)$ & $0.0(1.00)$ & $0.6(0.44)$ \\
\hline
\end{tabular}

Test for difference in allele frequencies between the two cohorts (Chi-square $=3.52, P=0.06)$ (N.B. totals for those with valid HP CNV genotype data but not necessarily overlapping other phenotypes).

was set at $(0.05 / 31=0.0016)$ and of the CaPS data was set at $(0.05 / 24=0.0021)$.

\section{Results}

Table 1 summarises genotype and allele frequency data for the two cohorts.

Genotype and allele frequencies were similar in the two studies.

Table 2 shows the results of tests for association between haptoglobin genotype and vitamin $\mathrm{C}$ concentration, hemoglobin level, and CHD, for BWHHS and CaPS (from phase 2). Haptoglobin genotype is associated with hemoglobin level in BWHHS (hemoglobin concentration increasing with number of $\mathrm{Hp} 2$ alleles) but not with $\mathrm{CHD}$ or vitamin $\mathrm{C}$ concentration. Haptoglobin genotype was not associated with coronary disease or hemoglobin concentration in CaPS. Though an association with haemoglobin was observed in BWHHS and not in CaPS, there was no statistical evidence that the association differed between these two studies $(P$ value from $z$-test $=0.24$ ).

\section{Validation Results}

Table 3 compares HP duplicon genotyping results using the assay of Koch et al. [11] and using our ratiometric assay (amplification ratio control system-ARCS). Genotyping was fully concordant between the two methods apart from Hp1,2 (96\% concordance).

The stated HP CNV genotypes of the control samples provided by Dr. Werner Koch and Kr. Joris Delanghe were in full agreement with those determined by ARCS (data not shown).

Figure 5 in [8] shows an ARCS plot of reference gene peak height on the $x$-axis against $H P$ gene $C N V$ peak height on the $y$-axis, for 85 samples. Hp1,1 Hp1,2, and Hp2,2 are seen to be separated into three distinct clusters radiating from the origin, providing good discrimination among the three genotypes.

Supplementary Data Tables 1 and 2 show the associations of haptoglobin genotype with phenome scan traits in BWHHS and in CaPS, respectively. Haptoglobin genotype was not associated with any phenotypes in either cohort, except for the association with hemoglobin in BWHHS as discussed above.

Supplementary Data Table 3 shows the haptoglobin genotype and allele frequencies by quartile of vitamin $\mathrm{C}$ distribution in BWHHS. No associations between genotype/allele frequency and vitamin $\mathrm{C}$ distribution were found.

\section{Discussion}

We examined the association between haptoglobin genotype and clinical traits in two British cohorts, with prior hypotheses for association with diabetic CHD, hemoglobin, and vitamin $\mathrm{C}$ level. Evidence of association with $\mathrm{CHD}$, diabetic $\mathrm{CHD}$, or vitamin $\mathrm{C}$ was lacking. Though haptoglobin genotype did show nominal association with hemoglobin, this was only in BWHHS and was not replicated in CaPS, though statistically the association was consistent between the two studies. Furthermore, despite the hypothesised antioxidant properties of haptoglobin and claims for the involvement of antioxidation in a wide range of health related outcomes, we found no compelling evidence that haptoglobin genotype was associated with a large number of phenotypes examined in our phenome scans (Supplementary Tables 1 and 2).

6.1. Haptoglobin Genotype and Vitamin C Status. We did not observe an association of haptoglobin genotype with plasma vitamin $\mathrm{C}$ concentrations. A small study of Chinese males $(N=50)[12]$ reported that individuals with Hp2,2-genotype had lower (by $31 \%$ ) vitamin C concentrations than those with other genotypes $(P<0.01)$ but stated that there was no such association in females $(N=60)$. It has also been reported [13] that overall haptoglobin concentrations are lower in males than females, resulting in lower stability of vitamin $\mathrm{C}$ in males. Additionally, Langlois et al. [6] noted that, in participants consuming a vitamin C-poor diet, those with the Hp2,2 genotype were most likely to develop vitamin $\mathrm{C}$ deficiency. Similarly, Cahill and El-Sohemy found that Hp2,2 participants had lower vitamin $\mathrm{C}$ concentrations when reported dietary vitamin $\mathrm{C}$ was low, but not when it met the recommended daily allowance [14]. These reports, together with the observations of Ness et al. [15] who showed a mean plasma vitamin C level in European British females of approximately 1.35 times the level found in males, suggest that our results for the entirely female BWHHS cohort may be a reflection of the vitamin $\mathrm{C}$ replete status of a large fraction of the participants [16]. When our data were divided into quartiles by vitamin C level (Supplementary Data Table 3) to test the hypothesis that genotype effect might only be apparent in low vitamin $\mathrm{C}$ quartiles, we also found no association.

6.2. Haptoglobin Genotype and Hemoglobin Levels. Our data suggest an association of haptoglobin genotype with hemoglobin level in BWHHS but not in CaPS. The difference in the sexes of the two cohorts may partially account for this result, since in several studies, hemoglobin concentration was found to be higher in males compared with females [17-19], but we found no statistical evidence that results did differ between the two cohorts. Alternatively, the lack of association 
TABLE 2: Association of HP CNV genotype with vitamin C and hemoglobin for BWHHS and CaPS cohorts.

\begin{tabular}{|c|c|c|c|c|c|}
\hline & & \multicolumn{3}{|c|}{ Means (SD) or $N(\%)$ of traits by genotype } & \multirow{2}{*}{$\begin{array}{c}P \text { trend } \\
(1 \mathrm{df})\end{array}$} \\
\hline & & Hp1,1 & $\mathrm{Hp} 1,2$ & Hp2,2 & \\
\hline \multicolumn{6}{|c|}{ Continuous outcomes: means (SD) } \\
\hline BWHHS & Vitamin C $(\mu \mathrm{mol} / \mathrm{L})$ & $43.53(27.47)$ & $44.03(28.75)$ & $42.91(28.26)$ & 0.51 \\
\hline CaPS & Vitamin C & $\mathrm{N} / \mathrm{A}$ & $\mathrm{N} / \mathrm{A}$ & $\mathrm{N} / \mathrm{A}$ & N/A \\
\hline BWHHS & $\mathrm{Hb}(\mathrm{g} / \mathrm{dL})$ & $13.45(1.00)$ & $13.49(1.14)$ & $13.61(1.00)$ & 0.002 \\
\hline CaPS & $\mathrm{Hb}(\mathrm{g} / \mathrm{dL})$ & $14.87(1.35)$ & $14.94(1.05)$ & $14.91(1.03)$ & 0.818 \\
\hline
\end{tabular}

Hb: hemoglobin.

TABLE 3: Comparison of HP duplicon genotyping results using Koch assay and using Amplification Ratio Control System (ARCS) assay for 64 data points.

\begin{tabular}{lcccc}
\hline & \multicolumn{4}{c}{ Hp CNV genotype } \\
& Hp1,1 & Hp1,2 & Hp2,2 & Fail \\
\hline Koch assay $(n)$ & 9 & 25 & 27 & 3 \\
ARCS assay $(n)$ & 9 & 24 & 27 & 4 \\
\hline
\end{tabular}

between haptoglobin genotype and hemoglobin concentration in the CaPS cohort may be a type-2 error. Prior biological knowledge of the high affinity specific interaction of haptoglobin with free hemoglobin (its main role) [20], together with a publication showing an association of the severity of anemia with haptoglobin genotype in patients infected with malaria [21] and on reporting an association of free hemoglobin level with haptoglobin genotype [12], led us to hypothesise that $H P$ would be associated with hemoglobin in the direction that we observed. Given the complex network of regulation and interactions, direction of effect is difficult to predict. We observe higher hemoglobin in the Hp2,2 group in BWHHS, contrasting with studies in malaria-endemic areas where Hp2,2 (additively) associates with lower hemoglobin levels in children during the malaria season and healthy Hpl,1 subjects display approximately $50 \%$ higher free hemoglobin levels in their plasma [12, 21].

Sex differences of iron turnover, as exemplified by protection of HFE gene homozygous mutant females from clinical hemochromatosis [22], the higher serum iron concentration found in Hp2,2 men but not Hp2,2 women, and the higher vitamin $\mathrm{C}$ concentration found in women [23] may all be important factors influencing effects of haptoglobin genotype.

In summary, our studies found no association between haptoglobin genotype and vitamin C status. An association was found between haptoglobin genotype and hemoglobin concentration in the BWHHS cohort (older women), but not in CaPS (middle-aged men). However, given the large number of associations examined here and some evidence of inconsistency of direction of association of $H P$ with hemoglobin in the literature, further replication of this finding is required before we can claim that this association is robust. A wider systems approach will be necessary to simultaneously resolve effects in relation to sex, redox status, free hemoglobin level, total hemoglobin level, and oxygen carriage, with potentially more major effects in the presence of large challenges to homeostasis such as those of malaria, diabetes, or other diseases.

\section{Conflict of Interests}

None of the authors had a conflict of interests.

\section{Authors' Contribution}

Authors' responsibilities were as follows. Philip A. I. Guthrie and Ian N. M. Day designed the research; Philip A. I. Guthrie conducted the research; John Gallacher, Debbie A. Lawlor, and Yoav Ben-Shlomo provided essential materials; Santiago Rodriguez and Debbie A. Lawlor analyzed data; Philip A. I. Guthrie wrote the paper; Santiago Rodriguez and Ian N. M. Day had primary responsibility for final content of the paper; all authors read and approved the paper.

\section{Acknowledgments}

The British Women's Heart and Health Study is supported by funding from the British Heart Foundation (BHF) and the Department of Health Policy Research Programme (England). The Caerphilly Prospective study was funded by intramural support from the MRC as part of the MRC Epidemiology Unit (South Wales). The MRC unit data archive is under the custodianship of the School of Social and Community Medicine. The MRC CAiTE Centre receives funds from the UK Medical Research Council (G0600705) and University of Bristol. The work done by PG is supported by the MRC (G0600705). This work was also supported by the MRC project Grant MR/K002767/1.

\section{References}

[1] N. Maeda, F. Yang, and D. R. Barnett, "Duplication within the haptoglobin Hp2 gene,” Nature, vol. 309, no. 5964, pp. 131-135, 1984.

[2] L. Garby and W. D. Noyesv, "Studies on hemoglobin metabolism. I. The kinetic properties of the plasma hemoglobin pool in normal man," The Journal of Clinical Investigation, vol. 38, pp. 1479-1483, 1959.

[3] P. Galan, S. Briançon, A. Favier et al., "Antioxidant status and risk of cancer in the SU.VI.MAX study: is the effect of supplementation dependent on baseline levels?" British Journal of Nutrition, vol. 94, no. 1, pp. 125-132, 2005. 
[4] T. Okazaki, Y. Yanagisawa, and T. Nagai, "Analysis of the affinity of each haptoglobin polymer for hemoglobin by twodimensional affinity electrophoresis," Clinica Chimica Acta, vol. 258, no. 2, pp. 137-144, 1997.

[5] M. Kristiansen, J. H. Graversen, C. Jacobsen et al., "Identification of the haemoglobin scavenger receptor," Nature, vol. 409, no. 6817, pp. 198-201, 2001.

[6] M. R. Langlois, J. R. Delanghe, M. L. de Buyzere, D. R. Bernard, and J. Ouyang, "Effect of haptoglobin on the metabolism of vitamin C," The American Journal of Clinical Nutrition, vol. 66, no. 3, pp. 606-610, 1997.

[7] S. H. Atkinson, T. W. Mwangi, S. M. Uyoga et al., "The haptoglobin 2-2 genotype is associated with a reduced incidence of Plasmodium falciparum malaria in children on the Coast of Kenya," Clinical Infectious Diseases, vol. 44, no. 6, pp. 802-809, 2007.

[8] P. A. I. Guthrie, T. R. Gaunt, M. R. Abdollahi et al., "Amplification ratio control system for copy number variation genotyping," Nucleic Acids Research, vol. 39, no. 8, article e54, 2011.

[9] D. A. Lawlor, C. Bedford, M. Taylor, and S. Ebrahim, "Geographical variation in cardiovascular disease, risk factors, and their control in older women: British Women's Heart and Health Study," Journal of Epidemiology and Community Health, vol. 57, no. 2, pp. 134-140, 2003.

[10] P. C. Elwood, "Caerphilly and Speedwell collaborative heart disease studies," Journal of Epidemiology and Community Health, vol. 38, no. 3, pp. 259-262, 1984.

[11] W. Koch, W. Latz, M. Eichinger et al., "Genotyping of the common haptoglobin Hp 1/2 polymorphism based on PCR," Clinical Chemistry, vol. 48, no. 9, pp. 1377-1382, 2002.

[12] N. Na, J. R. Delanghe, Y. E. C. Taes, M. Torck, W. R. G. Baeyens, and J. Ouyang, "Serum vitamin C concentration is influenced by haptoglobin polymorphism and iron status in Chinese," Clinica Chimica Acta, vol. 365, no. 1-2, pp. 319-324, 2006.

[13] J. Schrijver, H. J. M. Van Rijn, and W. H. P. Schreurs, "Reevaluation of the haptoglobin reference values with the radial immunodiffusion technique," Clinical Biochemistry, vol. 17, no. 4, pp. 258-262, 1984.

[14] L. E. Cahill and A. El-Sohemy, "Haptoglobin genotype modifies the association between dietary vitamin $\mathrm{C}$ and serum ascorbic acid deficiency," The American Journal of Clinical Nutrition, vol. 92, no. 6, pp. 1494-1500, 2010.

[15] A. R. Ness, F. P. Cappuccio, R. W. Atkinson, K.-T. Khaw, and D. G. Cook, "Plasma vitamin C levels in men and women from different ethnic backgrounds living in England," International Journal of Epidemiology, vol. 28, no. 3, pp. 450-455, 1999.

[16] A. J. Michels and B. Frei, "Myths, artifacts, and fatal flaws: identifying limitations and opportunities in vitamin C research," Nutrients, vol. 5, no. 12, pp. 5161-5192, 2013.

[17] S. Saxena and E. T. Wong, "Heterogeneity of common hematologic parameters among racial, ethnic, and gender subgroups," Archives of Pathology and Laboratory Medicine, vol. 114, no. 7, pp. 715-719, 1990.

[18] W. W. Hawkins, V. G. Leonard, and E. Speck, "The total body hemoglobin in children and its relation to caloric and iron requirements," Metabolism, vol. 5, no. 1, pp. 70-78, 1956.

[19] E. Humpeler and H. Amor, "Sex differences in the oxygen affinity of hemoglobin," Pflugers Archiv European Journal of Physiology, vol. 343, no. 2, pp. 151-156, 1973.

[20] M. R. Langlois and J. R. Delanghe, "Biological and clinical significance of haptoglobin polymorphism in humans," Clinical Chemistry, vol. 42, no. 10, pp. 1589-1600, 1996.
[21] H. Imrie, F. J. I. Fowkes, P. Michon et al., "Haptoglobin levels are associated with haptoglobin genotype and $\alpha+$-thalassemia in a malaria-endemic area," American Journal of Tropical Medicine and Hygiene, vol. 74, no. 6, pp. 965-971, 2006.

[22] R. Moirand, P. C. Adams, V. Bicheler, P. Brissot, and Y. Deugnier, "Clinical features of genetic hemochromatosis in women compared with men," Annals of Internal Medicine, vol. 127, no. 2, pp. 105-110, 1997.

[23] J. R. Delanghe and M. R. Langlois, "Haptoglobin polymorphism and body iron stores," Clinical Chemistry and Laboratory Medicine, vol. 40, no. 3, pp. 212-216, 2002. 


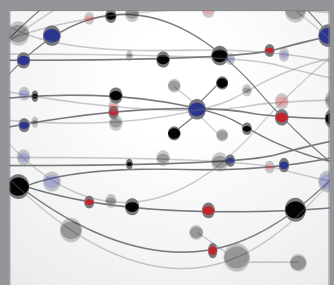

The Scientific World Journal
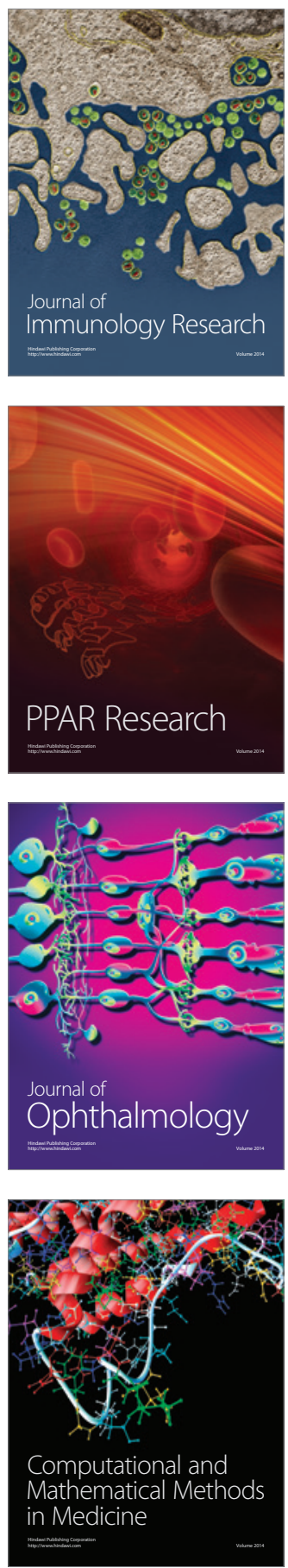

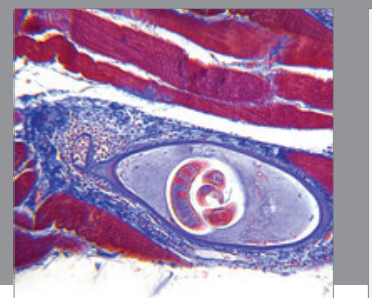

Gastroenterology

Research and Practice
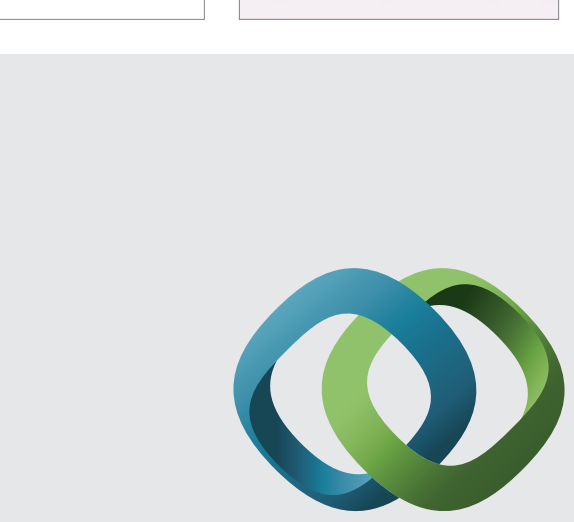

\section{Hindawi}

Submit your manuscripts at

http://www.hindawi.com
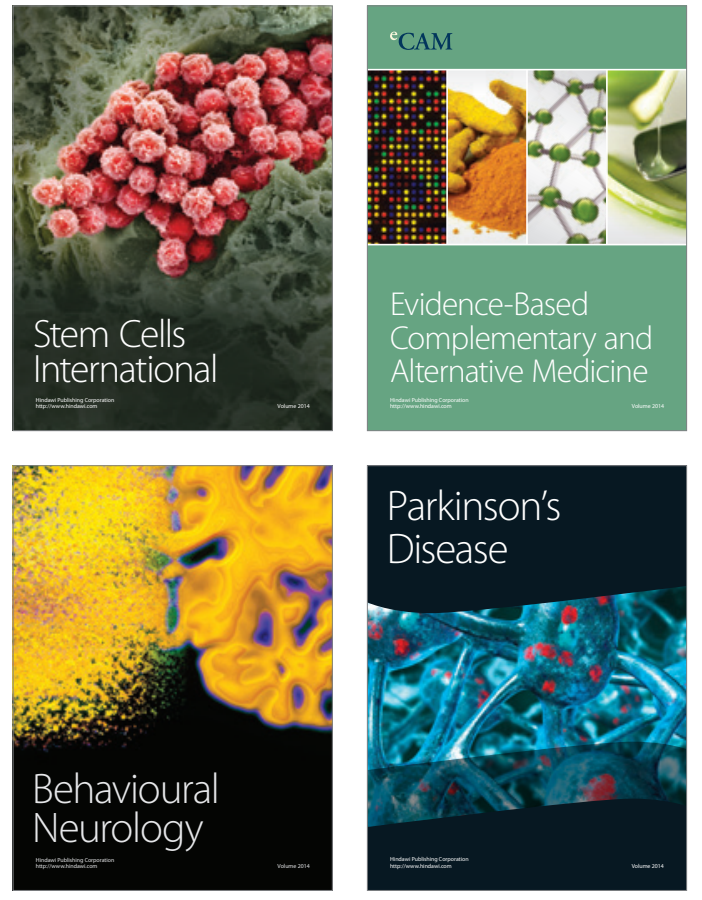
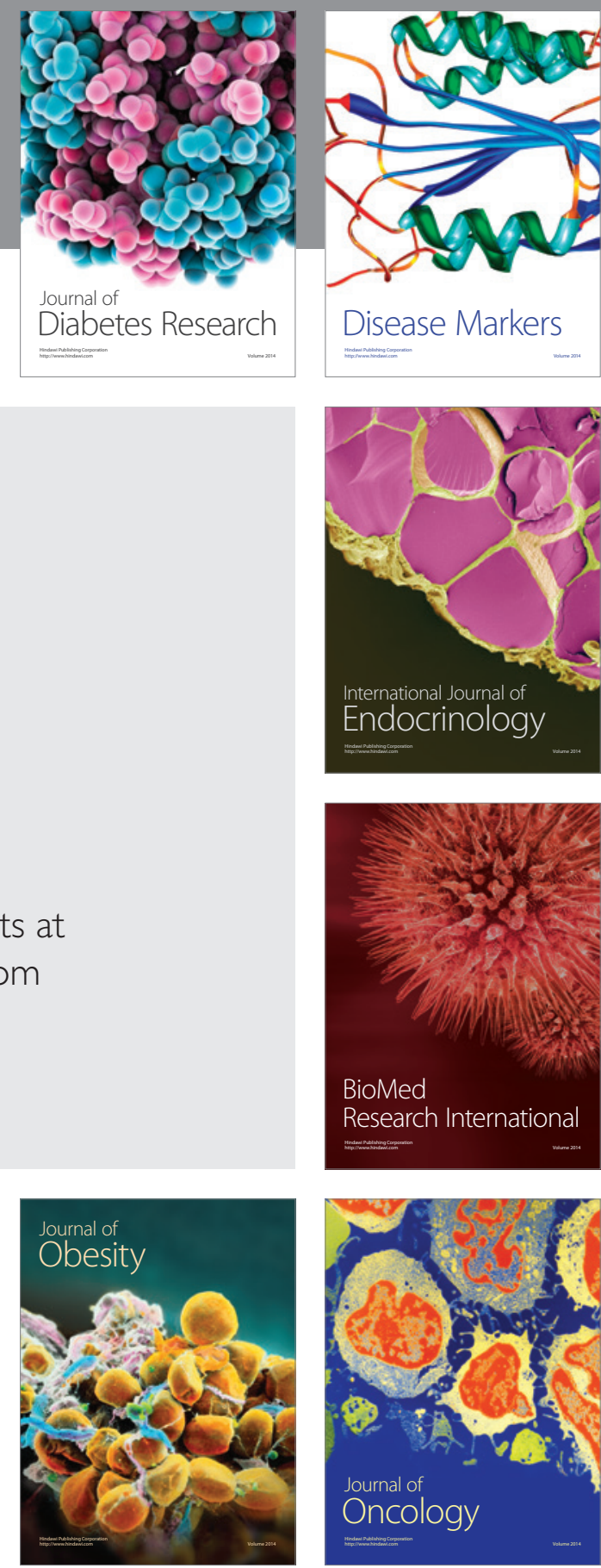

Disease Markers
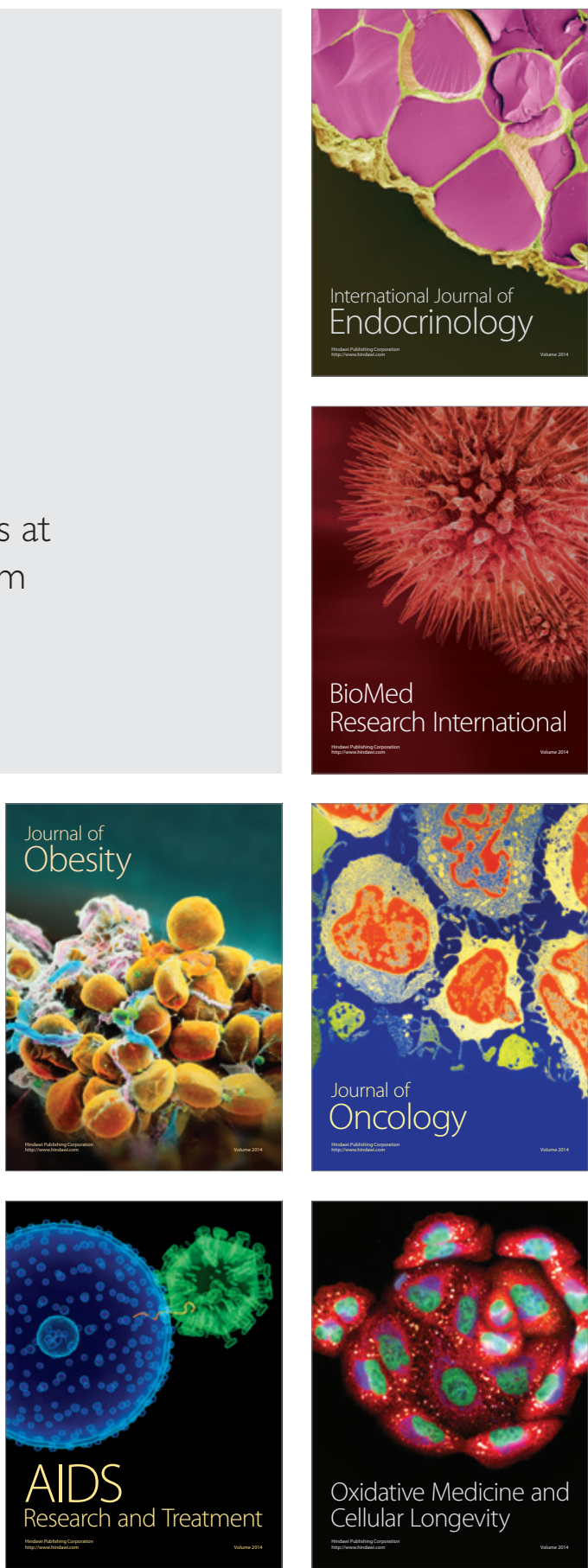